\section{A top-notch inhibitor}

\section{By Tim Fulmer, Senior Writer}

University of California, San Diego researchers have identified a role for Notch, a signaling pathway typically associated with cancer, in an entirely new indication-pulmonary arterial hypertension. ${ }^{1}$ In light of a new way to specifically inhibit Notch signaling described last week by a Harvard University team, ${ }^{2}$ this previously intractable pathway may now become a more realistic target for a wide range of indications.

Both sets of results are good news for Aileron Therapeutics Inc., which has licensed the Notch targeting platform from Harvard and could now design Notch inhibitors for multiple indications, including cancer and pulmonary disorders.

The four Notch receptors play a role in many developmental processes, and excessive activation of Notch signaling can trigger aberrant cellular proliferation. ${ }^{3}$ In particular, Notch signaling is important in the development of the vasculature and controls both proliferation of endothelial cells and differentiation of arteries and veins. ${ }^{4,5}$ One of the four Notch receptors, Notch homolog 3 (NOTCH3), has been implicated in regulating the development of vascular smooth muscle cells (VSMCs). ${ }^{6}$

Given those findings, a team at UCSD hypothesized that NOTCH3 might be involved in the development of $\mathrm{PAH}$, a disease characterized by excessive VSMC proliferation in vessel walls.

The researchers first looked at NOTCH3 activity in lung biopsies from $\mathrm{PAH}$ patients undergoing lung transplantation, from mice with hypoxia-induced PAH and from rats with chemically induced PAH. In all three systems, levels of NOTCH3 mRNA were higher in diseased lung tissue than those in tissue from the respective non-PAH controls.

Additional analysis of human and mouse lung tissue showed that NOTCH3 expression was confined to VSMCs of small pulmonary arteries.

The researchers then set about answering whether blocking NOTCH3 signaling could reduce or prevent PAH.

In mice, Notch 3 knockout prevented the development of hypoxic pulmonary hypertension such as occurred in wild-type littermates. The knockout animals had no medial thickening in the small pulmonary arteries and arterioles compared with controls.

Finally, wild-type mice with hypoxic pulmonary hypertension were treated with a daily dose of a $\gamma$-secretase inhibitor. The membrane-bound $\gamma$-secretase complex cleaves the Notch receptor to create the Notch intracellular domain (NICD), which translocates to the nucleus and activates Notch-targeted gene transcription. Inhibiting $\gamma$-secretase thus blocks Notch signaling (see Figure 1, "New findings in Notch signaling").

In the experiment, the $\gamma$-secretase inhibitor reduced thickening of vessel walls and decreased pulmonary hypertension compared with vehicle.

The researchers concluded that "inhibition of the expression or effect of NOTCH3 signaling in the adult pulmonary vasculature may be a useful strategy to prevent and treat PAH in humans."

The findings were published in Nature Medicine.

\section{One notch at a time}

Corresponding author Patricia Thistlethwaite told SciBX that NOTCH3 inhibitors could be disease modifying, in contrast to vasodilators that are marketed to treat $\mathrm{PAH}$. "Blocking NOTCH3 potentially prevents or reverses VSMC proliferation that leads to vessel wall thickening in later stages of disease," said Thistlethwaite, who is an associate professor of surgery at the University of California, San Diego School of Medicine.

Vasodilators primarily work in the early stages of PAH and are not disease modifying, she said.

Researchers contacted by SciBX had mixed opinions on whether repurposing $\gamma$-secretase inhibitors to block Notch signaling in PAH would be a good idea.

Small molecule $\gamma$-secretase inhibitors in the clinic include Merck \& Co. Inc.'s MK-0752, which is in Phase I/II testing to treat breast cancer, Eli Lilly and Co.'s LY450139, which is in Phase III testing for Alzheimer's disease, and Pfizer Inc.'s begacestat, which is in Phase I for AD.

Adrian Harris, professor of oncology at University of Oxford and director of Cancer Research U.K.'s Molecular Oncology Laboratories at The Weatherall Institute of Molecular Medicine at University of Oxford, thinks the molecules are not well suited to PAH because of their association with gut toxicity. In addition, he said "more than 40 cell-surface proteins are cleaved by the $\gamma$-secretase complex, so blocking that enzyme could have undesirable, broad-spectrum effects."

The $\gamma$-secretase inhibitor used in the Nature Medicine article, a research reagent called $N$-[N-(3,5-difluorophenacetyl)-L-alanyl]$S$-phenylglycine t-butyl ester (DAPT), produced no GI side effects in the mice at daily doses of $10 \mathrm{mg} / \mathrm{kg}$ for 15-25 days.

"That dose is lower than what's been used in the cancer clinic, where GI toxicity can be a problem. So if you can get away with a lower dose and see a treatment effect, $\gamma$-secretase inhibitors may still be an option in PAH," said Urban Lendahl, a professor of genetics at the Karolinska Institute whose research is focused on the interaction between Notch signaling and the cellular hypoxic response. ${ }^{7}$

"It's important to realize that dosing regimens for $\gamma$-secretase inhibitors have been developed to help get around the toxicity issue 
Figure 1. New findings in Notch signaling. Separate papers have identified a new indication, pulmonary arterial hypertension, associated with Notch and a way to actually target the previously intractable pathway.

At the cell surface, the Notch receptor ligand binds to the transmembrane Notch receptor [a]. This triggers a sequence of proteolytic cleavage events on the extracellular and intracellular side of the membrane [b]. The $\gamma$-secretase complex then cleaves an intracellular portion of the Notch receptor, generating the Notch intracellular domain (NICD).

Next, NICD translocates to the nucleus, in which it binds a transcription factor called CSL and a coactivator called mastermindlike 1 ( MAML1) to form a three-protein complex that activates genes [c]. In disease states, activation of Notch-targeting genes can trigger hyperproliferation that leads to cancer or vascular thickening that contributes to cardiovascular disorders like hypertension [d].

In a paper in Nature Medicine, researchers suggest that excessive activation of Notch homolog 3 (NOTCH3), which is one of the four Notch receptors, in the lung leads to hyperproliferation of vascular smooth muscle cells, which causes thickening of the vascular wall and $\mathrm{PAH}$. A paper published in Nature describes a peptide that blocks Notch signaling by inhibiting formation of the Notch transcription factor complex [c]. The peptide showed an antiproliferative effect in a rodent model of cancer.

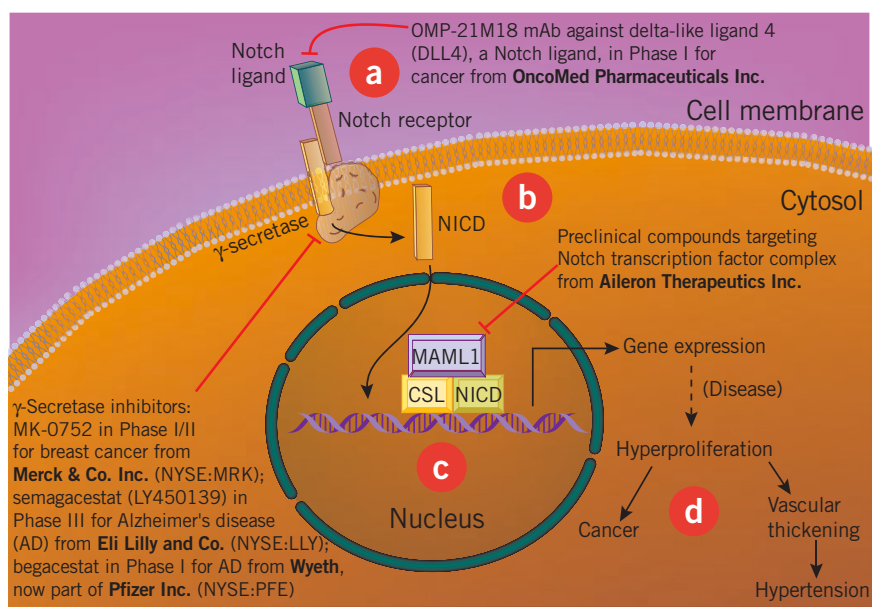

in cancer and Alzheimer's disease," he added. "Similar dosing regimens may also improve the safety of $\gamma$-secretase inhibitors in PAH. That work has yet to be done."

Ideally, said James Bradner, a strategy that selectively blocks NOTCH3 and leaves the other Notch receptors unaffected "would be expected to produce even fewer systemic side effects than targeting all the Notch receptors, as occurs with $\gamma$-secretase inhibitors."

Bradner is an investigator in the Department of Medical Oncology at Dana-Farber Cancer Institute and instructor of medicine at the Harvard Medical School.

Thistlethwaite agreed that going after NOTCH3 is probably the best approach. "Based on our findings, we believe the ideal $\mathrm{PAH}$ therapeutic would be an inhibitor specific for NOTCH3," she said.

Going forward, Thistlethwaite plans to study NOTCH3 signaling in rodent models of PAH. She said her team will focus on NOTCH3 target genes to determine if the proteins they encode influence other pathways implicated in VSMC proliferation. In the longer term, she hopes to look at NOTCH3 inhibitors in pig models of PAH.

Thistlethwaite said an invention disclosure covering her team's findings has been filed with the university's technology transfer office and is available for licensing.

\section{A notch further downstream}

Until last week, the idea of specifically targeting NOTCH3 - or any Notch receptor-seemed farfetched. Indeed, there are no disclosed small molecules or antibodies inhibiting individual Notch receptors in development. But a group led by Gregory Verdine of Harvard thinks it has hit upon a solution: peptide inhibitors that prevent formation of the Notch transcription complex in the nucleus.

The Harvard and Dana-Farber researchers reasoned that one way to reduce or avoid toxicity associated with blocking all Notch receptors would be to go downstream of the interaction between the cellsurface Notch receptor and $\gamma$-secretase. They opted to target Notch intracellular domain (NICD) derived from a single Notch family member with the goal of preventing NICD from interacting with prooncogenic target genes.

Inside the nucleus, NICD binds the transcription factor CSL and the coactivator mastermind-like 1 (MAML1) to form a three-protein complex that activates genes. A high-resolution X-ray crystal structure of the complex formed by the NOTCH1 NICD provided some initial guidance on potential

intervention points.

The crystal structure showed that a portion of MAML1 that bound NICD consisted of a nearly continuous stretch of $\alpha$-helices. Thus, the Harvard team hypothesized that a helical peptide mimetic might be sufficient to block binding of full-length MAML1 to NICD and thus inhibit transcriptional activation of Notch-targeted genes that drive cancer.

The researchers designed a series of six $\alpha$-helical peptides derived from MAML1. In vitro tests led to the selection of a 16-amino-acid peptide dubbed "stapled" $\alpha$-helical peptide derived from MAML1, or SAHM1.

Chemical crosslinks introduced into the $\alpha$-helices of SAHM1 modified its secondary structure and made it harder for proteases to recognize and degrade the peptide. Stapled peptides also are 
actively taken up by cells and are small enough to enter the nucleus, in which they can target transcriptional processes. ${ }^{8,9}$

A series of in vitro and cell culture studies confirmed that SAHM1 prevented MAML1 from binding to the NICD-CSL complex. As a result, the peptide blocked expression of NOTCH1 target genes and reduced proliferation of human $\mathrm{T}$ cell acute lymphoblastic leukemia (ALL) cell lines.

Finally, in a murine model of T cell ALL, twice-daily SAHM1 significantly reduced tumor burden compared with vehicle $(p=0.02)$.

The findings were published in Nature.

\section{Going forward}

Aileron Therapeutics has exclusively licensed the IP covering the stapled peptide platform. While negotiating a license for SAHM1, the biotech has optimized the stapled peptide to treat NOTCH1driven cancers, according to Verdine, a company cofounder and corresponding author on the Nature paper.

Verdine, a professor of chemistry at Harvard and director of the chemical biology program at Dana-Farber, said a priority for his lab will be to use the stapled peptide platform to design a series of peptides that are highly selective for each of the four NOTCH family members. Such selectivity should give the peptides a superior safety profile over other Notch-targeting compounds like $\gamma$-secretase inhibitors, he said.

He also noted that the platform does not depend on X-ray crystal structures.

"Certainly having an X-ray crystal structure available accelerates the design process. However, it is not a requirement," said Verdine. "Simply starting from an available amino acid sequence, one can rapidly synthesize a small library of stapled $\alpha$-helical peptides and screen them for inhibitory activity."

Selective inhibitors could be useful outside of cancer. "There are other roles for SAHM1. The peptide could be useful for treating other conditions characterized by $\mathrm{T}$ cell-mediated hyperproliferation, including organ rejection, graft-versus-host disease, autoimmunity and aplastic anemia," said Bradner, who also was a corresponding author on the Nature paper.

Bradner said he saw no reason why the stapled peptide described in Nature couldn't be further optimized to selectively target NOTCH3 and thus be used to treat PAH.

Indeed, Aileron already is looking past cancer.
"Going forward, the company has multiple preclinical programs that are focused on using the stapled peptide platform to design peptides that target transcription factors and, more generally, protein-protein interactions," Huw Nash, VP of corporate development, told SciBX. "Besides oncology, we are looking at metabolic and inflammatory disorders."

"At the moment, the company's most advanced program is focused on designing stapled peptides that target the BCL-2 [B-cell lymphoma 2] family of apoptotic proteins to treat cancer and autoimmunity," said Nash. Those peptides have shown efficacy in vitro ${ }^{10}$ and in preclinical models of hematological cancers and solid tumors, he said.

Fulmer, T. SciBX 2(45); doi:10.1038/scibx.2009.1649

Published online Nov. 19, 2009

\section{REFERENCES}

1. Li, X. et al. Nat. Med.; published online Oct. 25, 2009; doi:10.1038/nm.2021

Contact: Patricia Thistlethwaite, University of California, San Diego, Calif.

e-mail: pthistlethwaite@ucsd.edu

2. Moellering, R. et al. Nature; published online Nov. 12, 2009; doi:10.1038/nature08543

Contact: James Bradner, Dana-Farber Cancer Institute, Boston, Mass.

e-mail: james_bradner@dfci.harvard.edu

Contact: Gregory Verdine, Harvard University, Cambridge, Mass. e-mail: gregory_verdine@harvard.edu

3. Bray, S. Nat. Rev. Mol. Cell Biol. 7, 678-689 (2006)

4. Hofmann, J. \& Iruela-Arispe, M. Circ. Res. 100, 1556-1568 (2007)

5. Sainson, R. \& Harris, A. Angiogenesis 11, 41-51 (2008)

6. Roca, C. \& Adams, R. Genes Dev. 21, 2511-2524 (2007)

7. Lendahl, U. et al. Nat. Rev. Genet.; published online Nov. 3, 2009; doi:10.1038/nrg2665

8. Wolfson, W. Chem. Biol. 16, 910-912 (2009)

9. Sawyer, T. Chem. Biol. Drug. Des. 73, 3-6 (2009)

10. Gavathiotis, E. et al. Nature 455, 1076-1081 (2008)

\section{COMPANIES AND INSTITUTIONS MENTIONED}

Aileron Therapeutics Inc., Cambridge, Mass.

Dana-Farber Cancer Institute, Boston, Mass.

Eli Lilly and Co. (NYSE:LLY), Indianapolis, Ind.

Harvard Medical School, Boston, Mass.

Harvard University, Cambridge, Mass.

Karolinska Institute, Stockholm, Sweden

Merck \& Co. Inc. (NYSE:MRK), Whitehouse Station, N.J.

Pfizer Inc. (NYSE:PFE), New York, N.Y.

University of California, San Diego, Calif.

University of California, San Diego School of Medicine,

San Diego, Calif.

University of Oxford, Oxford, U.K.

The Weatherall Institute of Molecular Medicine at University of Oxford, Oxford, U.K. 